\title{
SENTIDOS DE LA BIOÉTICA QUE EMERGEN EN UN CONTEXTO INTERCULTURAL: UNA APROXIMACIÓN DESDE LA TEORÍA FUNDAMENTADA
}

\author{
Adriana Lucía Valdez Fernández ${ }^{1}$ y Francisco Fernando Bohórquez Gongora ${ }^{2}$ \\ Universidad del Cauca, Colombia. 1adrianitalvf@unicauca.edu.co; ²fbohorquez@unicauca.edu.co
}

\begin{abstract}
Resumen. Este estudio se centra en la formación de futuros profesionales de enfermería con el fin de conocer cómo se vive la formación bioética en un contexto intercultural como lo es el departamento del Cauca en Colombia. Se contó con la participación de docentes y estudiantes de la Universidad del Cauca, además de profesionales que laboran en el Hospital Mama Dominga del municipio de Silvia. Para esto se realizó un diseño cualitativo, enfoque epistémico la hermenéutica y análisis por medio de la teoría fundamentada. La información fue obtenida por medio de la revisión documental, observación participante, entrevistas individuales y grupales (grupo focal). Se encontró que, aunque los documentos plantean una apertura epistémica y ontológica hacía una visión que considere al ser humano, su cultura y su contexto, partiendo desde perspectivas bioéticas e interculturales, los discursos en la práctica muestran que hay una colonización del ser y el conocer en la formación donde se sigue privilegiando solo el saber técnico y biológico. Los mismos actores han planteado desde sus discursos formas de superar esta problemática, centrándose principalmente en la reflexión pedagógica como punto de partida para resignificar el currículo.
\end{abstract}

Palabras clave: Educación en Enfermería; Interculturalidad; Bioética; Teoría Fundamentada.

\section{SENSES OF BIOETHICS THAT EMERGE IN AN INTERCULTURAL CONTEXT: AN APPROACH FROM GROUNDED THEORY}

\begin{abstract}
This study focuses on the education of future nursing professionals in order to know how bioethical training is experienced in an intercultural context such as the department of Cauca in Colombia. There were teachers and students from the Universidad del Cauca, as well as professionals working at the Mama Dominga Hospital in the town of Silvia. For this, a qualitative design, epistemic approach to hermeneutics and analysis was carried out through grounded theory. The information was obtained through documentary review, participant observation, individual and group interviews (focus group). It was found that, although the documents pose an epistemic and ontological opening towards a vision that considers the human being, its culture and its context, starting from bioethical and intercultural perspectives, the discourses in practice show that there is a colonization of being and knowing in training where only the technical and biological knowledge is privileged. The actors themselves have proposed ways to overcome this problem from their speeches, focusing mainly on pedagogical reflection as a starting point to resignify the curriculum.
\end{abstract}

Keywords: Education, Nursing; Interculturality; Bioethics; Grounded Theory.

\section{INTRODUCCIÓN}

La formación bioética de los futuros profesionales de enfermería se ubica en el problema de la colonialidad del saber y del ser (Mignolo, 2007). La colonialidad del saber entendida como un dominio de experiencia político, epistémico y subjetivo con un marcado sentido hegemónico y homogéneo; que reproduce la colonialidad en los espacios de formación institucional, donde el maestro se instaura como protagonista del saber y del hacer en una relación unidireccional. El discurso hegemónico influye sobre las instituciones prestadoras de salud quienes condicionan las prácticas de acuerdo a los mismos; lo que provoca la aparición 
de subjetividades construidas a partir de dispositivos de control (en relación con el saber y el poder), que se traducen en una perspectiva del conocimiento que no da cabida al diálogo compresivo y por tanto a la alteridad. En relación al estudiante, se produce un fenómeno de aculturación, donde se dificulta generar un aprendizaje significativo que permita articular teoría y práctica y generalizar sus conocimientos a escenarios de diversidad cultural, limitando sus posibilidades de actuación profesional en lógicas epistémicas distintas.

La colonialidad del ser, que supone la negación de la otredad. Se sustenta desde un modelo médico hegemónico (Menéndez, 2017), en enfermería esto conlleva a una visión de cuidado reactiva (Fawcett, 2005) que prioriza lo biológico sobre lo humano, el cual subvalora el sistema de creencias del otro, reduciendo el cuidado a la curación física. Esto provoca en el sujeto de cuidado insatisfacción, porque en la mayoría de los casos buscan un encuentro en el acto de cuidado más allá de diagnósticos y tratamientos (Jirwe, Gerrish, \& Emami, 2010). Por otro lado, la formación bioética hegemónica no desarrolla una visión amplia de la salud, limitando la enseñanza al aprendizaje de lo técnico e instrumental valido para determinado contexto, a la vez que excluye contextos alternos. Así, la bioética comienza a considerarse como una cátedra más del currículo, que no da paso a otras miradas en torno a la salud y limita la enseñanza a la aplicación de técnicas y conocimientos en una sola perspectiva, en cierto modo apartada del ejercicio práctico y profesional, cuidado despersonalizado al cual esta investigación pretende ofrecer horizontes de solución.

Ampliar el horizonte bioético de quien se dedica al cuidado de enfermería, adquiere gran importancia, pues convierte la experiencia del cuidado en un proceso genuino de interacción e interés por la cultura y la cosmovisión de los otros, hacia la construcción de una visión de mundo de enfermería que considera a la persona y su contexto: en una educación para la vida. Máxime teniendo en cuenta que Colombia es un país caracterizado por su diversidad étnica y cultural, situación aún más evidente en el departamento del Cauca, donde la distribución étnica es de $46.1 \%$ de mestizos con ascendientes indígena y blanco, $32.2 \%$ afros y $21.7 \%$ indígenas (DANE, 2014). Lo que implica al profesional en formación en sus escenarios de práctica enfrentarse a otras visiones de salud y de cuidado de la salud, desencadenando en choques entre el sujeto de cuidado y el cuidador, al no contar con la formación para asumir estos retos (Campinha-Bacote, 2015). Por lo cual se plantea la necesidad de una formación bioética de los futuros profesionales de enfermería donde la interculturalidad actúe como un puente en las relaciones que emergen en el proceso de aprendizaje de la bioética. 
Así, para De Souza (2013), la educación para la vida implica adscribirse al proyecto éticopolítico de la de-colonialidad, es decir, optar por una formación que surge de reconocer la existencia del "otro" socioculturalmente diferente, no al modo sajón de concebir las interrelaciones humanas, sino a la manera latinoamericana y particularmente signada por las interacciones entre culturas diferentes. El significado transformador de la interculturalidad en la formación bioética, se destaca en tanto prioriza la relación entre el yo, el otro y el contexto, lo cual equivale a vincular la singularidad personal, la otredad y lo universal, con un horizonte de sentido, abierto al cuestionamiento de la realidad y de la propia existencia. De donde surge la pregunta: ¿Cuáles son los sentidos de la bioética que emergen en la formación de profesionales de Enfermería de la Universidad del Cauca en un contexto intercultural? Teniendo en cuenta que los sentidos son el acercamiento a lo que experimenta una persona y cómo esto se relaciona al contexto, con los otros y consigo mismo (Holzapfel, 2005). El contexto intercultural al que se refiere la pregunta corresponde al hospital Mama Dominga del municipio de Silvia, Cauca, Colombia, en donde los estudiantes de enfermería realizan sus prácticas de formación con la población con que se relacionan, pertenecientes al grupo indígena Misak.

\section{METODOLOGÍA}

Para dar respuesta a la pregunta de investigación se sigue un enfoque cualitativo, en razón a que éste permite la comprensión de fenómenos individuales de carácter social, al tener en cuenta los significados y símbolos individuales que forman el mundo social, donde la intersubjetividad es clave para llevar a cabo las reflexiones necesarias que acerquen a la comprensión del fenómeno estudiado (Salgado, 2007). La elección de este enfoque, se produce por su carácter integrativo y holista que permite un acercamiento al ser humano reconociendo la subjetividad. Como señalan Blasco y Pérez "se encarga de realizar un estudio de la realidad del contexto natural, de cómo suceden los hechos y sometiendo a la interpretación esos fenómenos" (p. 25). En este sentido, el abordaje cualitativo desarrollado en la investigación permitió el reconocimiento de los discursos y narrativas de los participantes, desde la significación de experiencias y conceptos, posibilitando la emergencia de sentidos y su posterior interpretación desde el texto y el contexto.

Lo anterior, es de importancia en enfermería, al tener en cuenta que esta disciplina no solo atiende el componente biológico, sino también los aspectos psicosociales que constituyen la integridad de la persona, lo que implica considerar tanto el objeto físico de la intervención, 
como el contexto en que subyacen los rasgos culturales, así como las creencias, cosmovisión y representaciones construidas por los individuos y comunidades (sobre todo cuándo el cuidado se realiza en un contexto intercultural).

El tipo de estudio fue descriptivo, el cual de acuerdo con Niño (2011) tiene el propósito de "describir la realidad objeto de estudio, un aspecto de ella, sus clases, sus categorías o las relaciones que se pueden establecer entre varios objetos." (p. 34). De epistemología hermenéutica, la cual intenta develar el texto y su contexto, desde la mirada de quienes lo producen (Gadamer, 1993). Involucra "una actividad de reflexión en el sentido etimológico del término, es decir, una actividad interpretativa que permite la captación plena del sentido de los textos en los diferentes contextos por los que ha atravesado la humanidad." (Arraéz, Calles, \& Moreno, 2006, p. 174). Permite interpretar múltiples aspectos del fenómeno desde su contexto; evita emitir sentencias definitivas, y en lugar de ellos propone una interpretación rigurosa y permanente que se construye y deconstruye en la emergencia de sentidos. La hermenéutica surge como una necesidad de inclusión de los problemas propios de la comprensión del espectador respecto a los objetos (Sánchez \& Sánchez, 2010). Los actores juegan un papel fundamental en la interpretación, considerando que el investigador debe "particularmente asumir la realidad como construida y asumir que el otro, el sujeto, es un constructor válido de la realidad y no una fuente de provisión de datos" (Sánchez, 2012, p. 88). Así, hacer hermenéutica implica "entender al interlocutor desde su perspectiva y situación" (Sánchez \& Sánchez, 2010, p, 44).

En este caso particular, la hermenéutica permitió develar sentidos emergentes en los discursos de los actores de formación bioética (docentes, tutores de práctica, estudiantes y sujetos de cuidado), con relación a los sentidos encontrados en una revisión documental inicial respecto a cuatro categorías principales (Formación, Bioética, Enfermería e Interculturalidad); lo que posibilitó ampliar el horizonte de interpretación y llegar a la construcción de una categoría central (desde la teoría fundamentada). Como señalan Sánchez y Sánchez (2010), el término "horizonte" implica "extender las capacidades de comprensión al ampliar el eje temático, sin olvidar que la búsqueda de sentido hace parte de esas condiciones de una hermenéutica que empieza a buscar más allá de los limites aparentes. Encontrando y encontrándose". ( $p, 44)$. El análisis se realizó bajo la teoría fundamentada, en la búsqueda de generar o descubrir a través de los datos un modelo explicativo del objeto de estudio (Corbin \& Strauss, 2015). 


\subsection{Participantes}

La selección de los participantes (que corresponden a los actores de formación) se basó en criterios no probabilísticos según los objetivos de la investigación y las dos instituciones de interés: la Universidad del Cauca, de donde se seleccionaron tanto estudiantes como personas con vínculo laboral y el Hospital Mama Dominga, se tienen en cuenta a los profesionales que trabajan para dicho hospital y son responsables del acompañamiento de los estudiantes durante su práctica formativa. En total se incluyeron 33 personas, 14 docentes (cuatro asesores práctica, seis directivos del programa de enfermería, cuatro profesores relacionados con la formación bioética y procesos educativos, tanto de planta como ocasionales y jubilados); ocho estudiantes de último semestre del programa de enfermería y que realizarían su práctica formativa en un contexto de diversidad cultural en el municipio de Silvia, Cauca, de ellos seis eran de diferentes municipios del departamento y dos se encontraban en movilidad académica procedentes del departamento del Huila; seis tutores de práctica institucional ( 3 enfermeros, 2 médicos, 1 profesional salud ocupacional del hospital antes mencionado), los cuales son profesionales egresados de diferentes universidades y dos de ellos son miembros de la comunidad Misak, y cinco sujetos de cuidado Misak que fueron cuidados por los estudiantes.

\subsection{Técnicas e instrumentos de producción de datos}

Revisión documental. El primer paso fue la revisión de documentos desde el nivel macro a micro, relacionados con la formación bioética e interculturalidad, como lo fueron la Constitución Política de Colombia de 1991, la Ley general de educación (Ley 115 de 1994), la ley en salud relacionada con la formación del talento humano (Ley 1164 de 2007), la Ley por la cual se reglamenta la responsabilidad deontológica para el ejercicio de la profesión de enfermería en Colombia (Ley 911 de 2004), documentos de la Universidad del Cauca y del programa de enfermería, entre otros. Se describieron los conceptos y categorías principales, centrándose solo en los contenidos explícitos, con el fin de evitar errores de interpretación al no poder asegurar el sentido propio de los textos ni las intenciones de sus autores (Valencia, 2010).

Entrevistas semiestructuradas. Con el fin de conocer las opiniones de los participantes mediante la indagación oral se realizaron dos entrevistas a cuatro estudiantes, dos a cinco sujetos de cuidado, una a una tutora de práctica y cuatro a cuatro docentes, siendo un total de nueve entrevistas, la duración aproximada fue de entre 60 y 120 minutos cada una. Su 
forma de aplicación individual favorece la intimidad y libertad de expresión (Sánchez, Martín, \& Canal, 2017), para mantener a los participantes centrados en las categorías se llevaron a cabo de manera semiestructurada, partiendo de unas preguntas base que permitían al participante dar su opinión general con libertad, pero sin desviarse de la temática indagada. Las entrevistas además colaboraron al complementar el sentido encontrado en los documentos. Cada entrevista fue grabada y transcrita en su totalidad, garantizando la privacidad de los participantes usando códigos para identificarlos a cada uno.

Observación no participante. Para identificar las perspectivas de los participantes en la inmediatez y contexto se realizó observación no participante. Los acontecimientos fueron registrados en diarios de campo, con anotaciones, siempre asumiendo una posición pasiva para captar el sentido original que emergen de las situaciones. Los diarios de campo se llevaron a cabo en la inducción a la práctica tanto en la universidad como en el hospital, observación del desarrollo de la práctica por los estudiantes en consulta externa, actividades educativas con la comunidad y salidas extramurales (actividades fuera de la institución que hacen parte de sus labores y los acerca al contexto de los sujetos de cuidado), observación de cuatro visitas de seguimiento realizada por los asesores de práctica y observación de cierre de práctica y sustentación de proyectos, generando 17 diarios de campo registrados de forma narrativa en audios, videos y fotografías, transcritos textualmente para su posterior análisis.

Grupos focales. Se realizan con el fin de ir más allá del discurso de los participantes y conocer tanto sus percepciones como actitudes sobre las categorías. Estas no solo permiten profundizar en la información recolectada anteriormente, sino que llevan a los participantes a sentirse comprometidos con el estudio y los productos que surjan del mismo, asumiéndolos como sujetos activos, otorgándoles la oportunidad de contribuir, de decidir y de aportar como conocedores (Sánchez, Martín, \& Canal, 2017, p. 43). De este modo, se realizaron cinco entrevistas grupales, una a tutores de práctica, una a directivos y tres a estudiantes (dos al inicio del período de práctica y una al final del mismo). La información se registró en audios, escritos textualmente para su posterior análisis.

\section{ANÁLISIS DE LOS DATOS}

Se realizó en tres fases según la teoría fundamentada, teniendo en cuenta que se busca a través de los discursos conocer los sentidos de la bioética que emergen en la formación de los estudiantes en contextos interculturales, generando esquemas explicativos (figura 1). Esta metodología para el análisis posibilita que sea exhaustivo al generar una relación circular de 
los datos, donde no son abandonados para pasar a otro, sino que se oscila entre todos para ir extrayendo la información y construir una teoría a partir de ellos (Corbin \& Strauss, 2015).

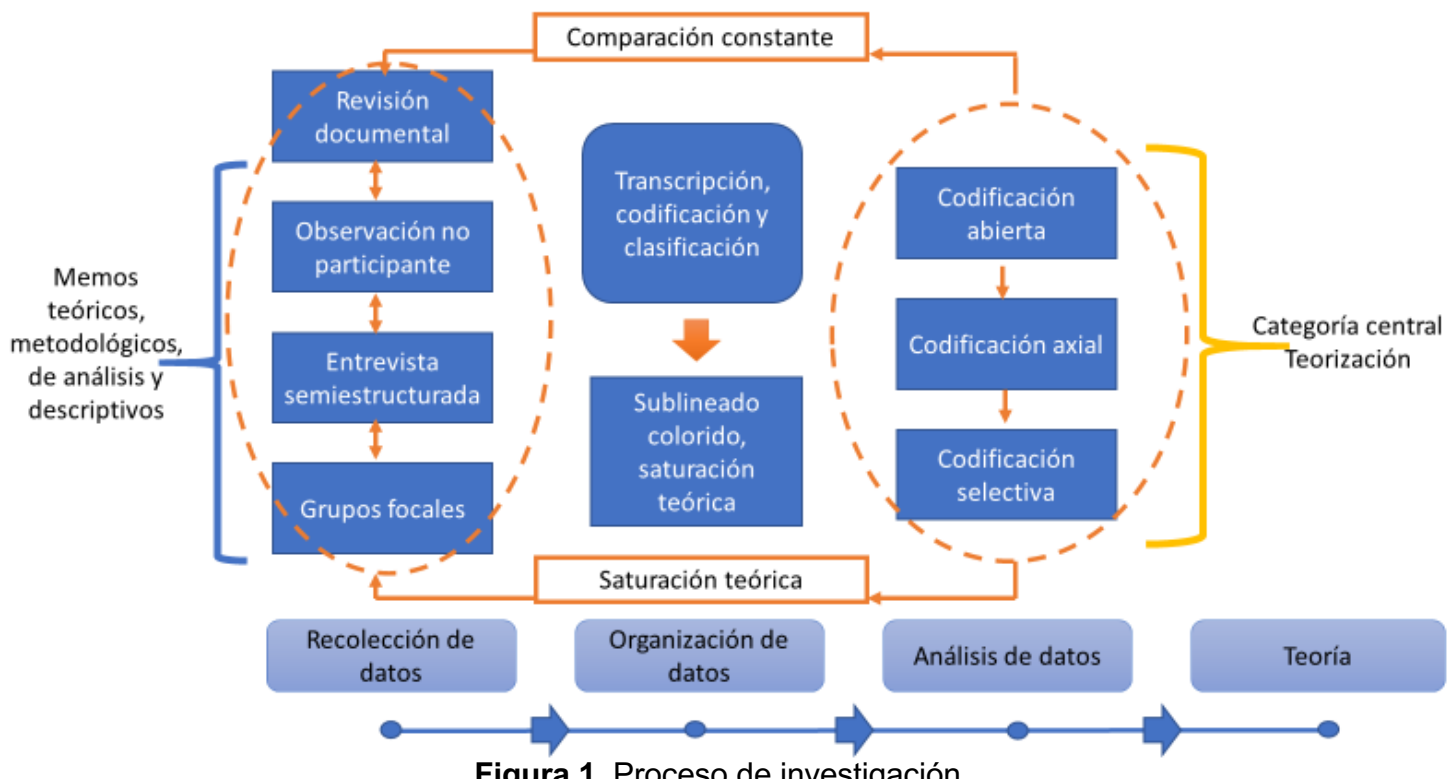

En la primera fase se realiza la recolección de datos, la cual se dividió en cuatro momentos: observación participante, revisión documental, entrevistas semiestructuradas y grupos focales. Se parte de cuatro categorías teóricas iniciales: práctica formativa, bioética, interculturalidad y enfermería, que guiaron la elaboración del análisis. La segunda fase se divide en codificación abierta y axial:

Codificación abierta. Se realizó un análisis detallado de los discursos examinados a través del microanálisis línea por línea, para esto la información fue transcrita y organizada, durante todo el proceso se realizaron memos para caracterizar la muestra (metodológicos), hacer un acercamiento a las relaciones y los códigos que se van a originar (teóricos), sintetizar análisis del investigador(analíticos) y describir observaciones durante el proceso de recolección de datos (descriptivos). Así, se logró descomponer los datos en códigos nominales, agrupándolos para posteriormente categorizarlos, lo cual fue posible por medio de la saturación teórica y la comparación constante, que señalaron el momento en que ya los datos no aportaban información adicional. Lo anterior por medio del sublineado colorido separando los temas e ideas centrales que definieron las categorías y subcategorías (Cuadro 1).

Cuadro 1. Categorías y subcategorías emergentes de la codificación abierta

\begin{tabular}{l|l} 
Categorías & Subcategorías
\end{tabular}




\begin{tabular}{|l|l|}
\hline Prácticas de formativas en la Bioética & $\begin{array}{l}\text { Currículo de formación bioética, Formación bioética, } \\
\text { para afrontar la vida, Practica: Educativa y Practica } \\
\text { Formativa. }\end{array}$ \\
\hline $\begin{array}{l}\text { Concepto de bioética en el campo de la } \\
\text { práctica profesional }\end{array}$ & $\begin{array}{l}\text { Estimación del concepto de Ética, Percepción de la } \\
\text { Bioética, con una aproximación a una tendencia } \\
\text { latinoamericana. }\end{array}$ \\
\hline $\begin{array}{l}\text { Interculturalidad para transformar la bioética } \\
\text { en el campo prácticas formativas }\end{array}$ & $\begin{array}{l}\text { Cultura, algo propio de la humano y que genera } \\
\text { identidad. } \\
\text { Interculturalidad, la mirada de uno mismo desde donde } \\
\text { uno mira al otro. }\end{array}$ \\
\hline $\begin{array}{l}\text { Visión del cuidado en enfermería para } \\
\text { transforma la bioética en las prácticas } \\
\text { formativas en un contexto intercultural }\end{array}$ & Persona, contexto, cuidado y salud. \\
\hline
\end{tabular}

Codificación axial. Una vez se tiene la categorización analítica se buscaron las relaciones entre las categorías y subcategorías que se encontraron en la codificación abierta (Strauss \& Corbin, 2002). Para esto, se contextualizaron los hallazgos teniendo en cuenta seis preguntas propuestas por Strauss y Corbin (2002): ¿Cuáles son las condiciones causales?; ¿Cuál es el fenómeno?; ¿Cómo es el contexto?; ¿Qué condiciones intermedias se presentan? ¿Cuáles son las estrategias? y ¿Cuáles son las consecuencias? Nutriendo la descripción de las categorías y permitiendo establecer apropiadamente las relaciones entre ella, de tal manera que las variables centrales son definidas según los vínculos que se distinguieron.

En la tercera fase, codificación selectiva, se vislumbran los núcleos temáticos donde se realiza el "proceso de integrar y refinar la teoría." (Strauss \& Corbin, 2002. p. 157). Para esto se organizaron las variables y categorías en torno a un concepto explicativo central (figura 2), a partir de las concepciones sobre el fenómeno estudiado (Strauss \& Corbin, 2002). Así, la teorización corresponde a la explicación principal que se aportó como investigador, con base a la condensación de las relaciones de las categorías y subcategorías, como también de las variables centrales. 


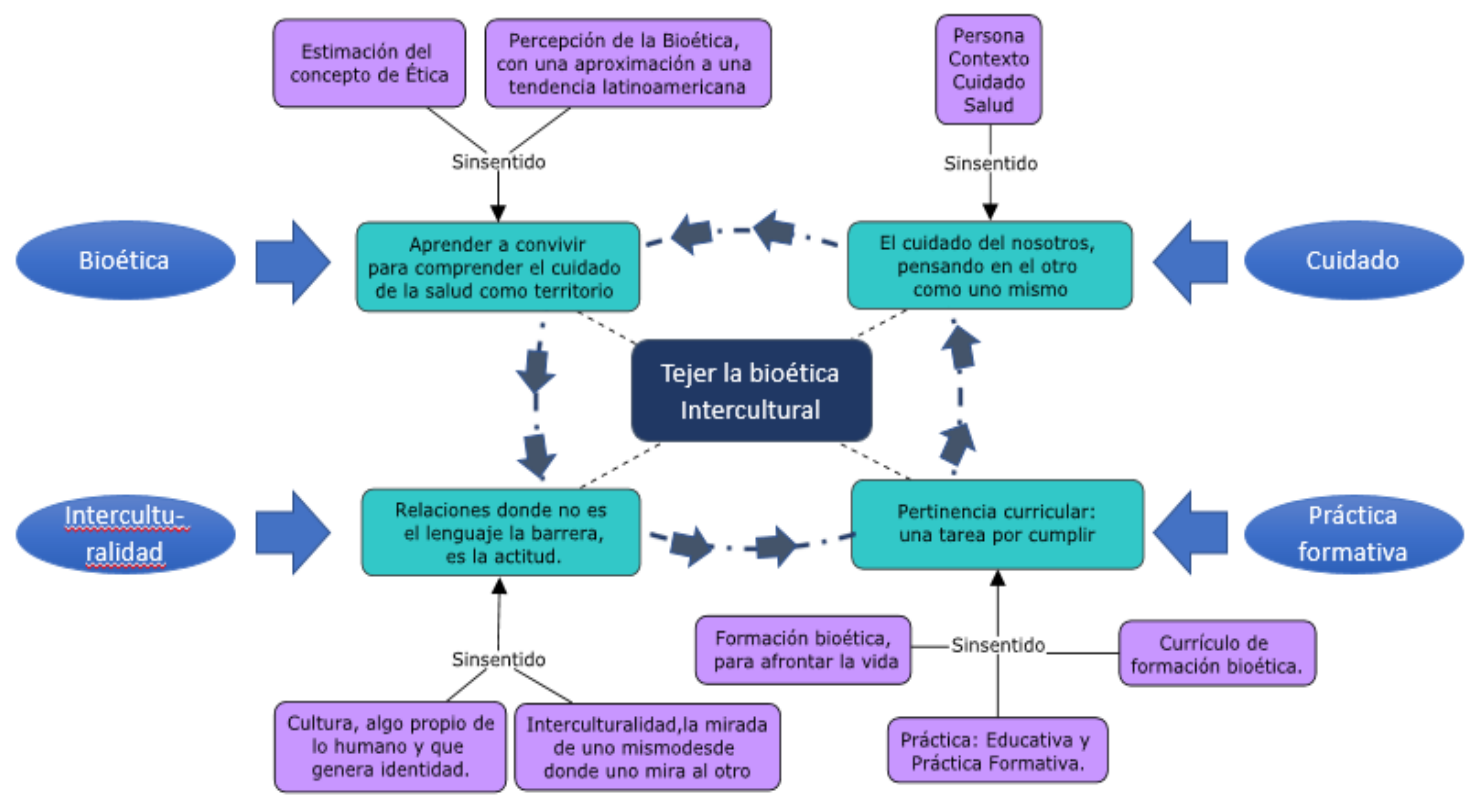

Figura 2. Categoría central y las relaciones en las que emerge.

\section{DISCUSIÓN Y RESULTADOS}

Se exponen las categorías en relación a los sinsentidos planteados entre los documentos y los discursos de los actores de formación, los cuales dan paso a las variables centrales. Cada categoría, se presenta por separado lo cual no significa que no se teje a partir de su interrelación con la otra. Así, emerge la categoría central, tratada en la conclusión, como una forma de condensar la información y hallazgos.

\subsection{Categorías}

Prácticas formativas en la bioética. Referente al análisis realizado en los documentos, se encontró que en papel se busca que los estudiantes sean formados para un desarrollo integral, que incluye formar en autonomía, capacidad de toma de decisiones, pensamiento crítico, actitudes éticas e inteligencia emocional y social, "formación integral del ser humano, como un proceso permanente y participativo que busca desarrollar todas sus dimensiones: ética, espiritual, cognitiva, afectiva, comunicativa, estética, corporal y socio-política, a fin de lograr su realización plena en la sociedad" (Documento institucional Proyecto Educativo de Enfermería, 2018). Sin embargo, desde los actores de formación, se presenta un currículo enfocado en contenidos conceptuales y técnicos que limita al estudiante en la adquisición de conocimientos necesarios para responder a las exigencias de la práctica. Se muestra entonces un problema en la pertinencia curricular estableciéndose como una tarea por 
cumplir: "Para hacer, usted no necesita pensar, usted no necesita analizar, usted no necesita criticar, justed tiene que hacer!, y lograr que le pongan cinco (refiriéndose a la calificación máxima en Colombia)" (Docente de Enfermería 7, entrevista, 12 de junio de 2018). Situación que afecta la visión bioética en la formación.

Concepto de bioética en el campo de la práctica profesional. Los documentos plantean una visión de bioética centrada en principios éticos y morales, que propenden por el respeto a los derechos humanos y guían el actuar de los profesionales en la toma de decisiones cuando se enfrentan a dilemas éticos de base cultural. "El Talento Humano del área de la Salud se regirá por los siguientes principios generales: ética. La formación y el desempeño del Talento Humano en Salud, debe estar enmarcado en el contexto cuidadoso de la vida y la dignidad del ser humano" (Documento nacional Talento Humano en salud, 2007). A pesar de que esta categoría ha ido evolucionando en el discurso, en la práctica se dista del concepto en razón a que desde la comunidad Misak hay una visión de salud distinta que amplía el campo de reflexión de la bioética, mencionan que "salud para nosotros va más allá de solo mirar la enfermedad; es pensar en el territorio [...] es lo que vivenciamos diariamente, lo que nos han enseñado. $Y$ a partir de esto nosotros podemos prevenir todo, por eso podemos hablar de salud mental, de salud sexual y reproductiva, de salud de todo" (Profesional Hospital Mama Dominga, intervención en grupo focal, 10 de noviembre de 2017). Aunado a un proceso formativo que no da cuenta del análisis de cómo el respeto por la cosmovisión en torno al cuidado de la salud permea la toma de decisiones en el ejercicio profesional. Lo que se ha evidenciado en cambios de comportamiento de los estudiantes dependiendo del contexto de práctica porque los aprendizajes que han tenido con respecto a la bioética han quedado atrás y quienes los forman reconocen que no cuentan con bases epistémicas. "El temor de hablar sobre ética, por el desconocimiento de la rama" (Docente 6, entrevista, 12 de junio de 2018). Implicaría entonces reconocer que aprender a convivir, requiere la participación de todos los actores de formación en dotar al estudiante de capacidad en la toma de decisiones para comprender el cuidado de la salud como territorio.

Interculturalidad para transformar la bioética en el campo de las prácticas formativas. Desde los documentos se reconoce la diversidad étnica y cultural, así como el conocimiento de la cultura del otro y la de uno mismo. "la educación se desarrollará atendiendo a los siguientes fines: El estudio y la comprensión crítica de la cultura nacional y de la diversidad étnica y cultural del país, como fundamento de la unidad nacional y de su identidad" (Documento nacional de educación, 1996). No obstante, en la práctica, la formación se reduce 
al desarrollo de competencias en las relaciones y el reconocimiento del otro diverso como una estrategia de inclusión que mantiene la asimetría de las relaciones en el acto de cuidado. El contexto demanda trascender hacia una interculturalidad que actúe como puente entre lo propio y lo externo, es decir una articulación para que la formación bioética integre la cosmovisión de las personas en torno al cuidado de la salud con los conocimientos adquiridos durante el desarrollo de la formación del futuro profesional en enfermería. "Articular ambas culturas de manera que se logre el mayor beneficio para la población sin tener que llegar a choques culturales sino a un acuerdo" (Estudiante de Enfermería 4, intervención en grupo focal, 15 de noviembre de 2017). Se busca la preservación cultural y el respeto por la autonomía de las personas al momento de brindar el cuidado en enfermería, donde la comunicación es el eje fundamental para establecer una relación con el otro, que indica Relaciones donde no es el lenguaje la barrera, es la actitud.

\section{Visión de cuidado en enfermería para transformar la bioética en las prácticas formativas} en un contexto intercultural. Los documentos priorizan la formación epistémica y ontológica de los futuros profesionales, reconociendo el carácter holístico del ser y enfatizando en la formación de pensamiento crítico para realizar un cuidado contextualizado y responsable: "EI cuidado de salud propia e intercultural hace referencia a las acciones en salud propia y complementaria que se realizan con las personas, las familias, la comunidad y con el territorio, que tiendan a promover y proteger la salud, y a prevenir y tratar la enfermedad con el propósito de promover, recuperar y mantener la armonía y el equilibrio" (Documento nacional lineamientos de incorporación del enfoque intercultural en la formación del talento humano en salud de 2017). No obstante, los documentos institucionales no hacen explicita la visión ontológica y epistémica del cuidado que orientan la formación bioética. Desde la voz de los actores se demanda una visión de cuidado reciproca que reclama no solamente orientación ontológica y epistémica desde el terreno racional, sino experiencias de aprendizaje durante las prácticas formativas que propendan por una relación dialógica del reconocimiento del otro de su saber y su responsabilidad en torno al cuidado de su salud en la búsqueda de denominadores comunes. "Te pones en la posición de la otra persona y cuidas desde la percepción de la otra persona, [desde lo que necesita], no imponiendo lo que sabes" (Estudiante de Enfermería 1, diario de campo, 29 de septiembre de 2017). Es decir, el Cuidado del nosotros, pensando en el otro como uno mismo. 


\subsection{Variables centrales}

Pertinencia curricular, una tarea por cumplir. Se requiere superar la mirada biologicista que se vive en la formación bioética de profesionales de enfermería, en razón a que la homogenización va en contra del mismo espíritu universitario que defiende la libertad, fomentando el respeto e inclusión de la diferencia (De Zubiría, 2016). Para cumplir esto, se requiere del diálogo entre la universidad y el contexto, que desde una perspectiva flexible empiece a de-colonizar el saber, en un currículo que se construya desde el contexto en razón a sus necesidades y saberes.

Aprender a convivir para comprender el cuidado de la salud como territorio. La bioética se concibe como un concepto inacabado y que se construye en experiencias de aprendizaje en los contextos de práctica, en la interacción con los otros en el acto de cuidado, donde emergen relaciones y choques que conllevan cuestionamientos sobre el quehacer profesional, las visiones culturales y la formación recibida. Los actores de formación reclaman que se les dé a conocer otras miradas del ser y conocer, con el fin de abandonar prejuicios y contextualizarse sobre los espacios donde se realizan las prácticas. Esto permite el decolonizar el ser de los estudiantes, lo que les dota de apertura, que posibilita un mejor acercamiento con el otro diverso y cuyo inicio es la de-colonización del saber de los docentes.

Relaciones donde no es el lenguaje la barrera, es la actitud. La formación bioética en los contextos de práctica diversos culturalmente emerge en las relaciones que se construyen tanto en el acto pedagógico como en el acto de cuidado, ya sea entre el docente y estudiante o entre el cuidador y el sujeto de cuidado. Se evidencia que en este encuentro el lenguaje no es la barrera, es la actitud bajo la cual se ha formado el futuro profesional al carecer de una actitud de escucha que propenda por comprender al otro. Se reclama la interculturalidad como un puente para la transformación de la formación bioética, no solo basta con reconocer la diversidad cultural del otro o aprender su lenguaje para relacionarse, sino una formación en que busque denominadores comunes que conlleven a relaciones de apertura a otras miradas del cuidado de la salud.

Cuidado del nosotros, pensando en el otro como uno mismo. Se requiere reconfigurar la pedagogía, lo curricular y lo disciplinar hacia una visión más humana del cuidado para deconstruir la barrera de formación bioética que está en nosotros mismos. Así, el futuro profesional se siente parte del contexto, lo cual es posible en una formación desde una visión crítica que le concedan abstraer sentidos de las relaciones que emergen en el acto de cuidado y le ayuden a construir una visión más humana. Se exige entonces del docente un papel 
activo, desde la pedagogía del conocer y del vivir, que enseñe desde su experiencia como ejemplo de formación bioética y considere a sus estudiantes como iguales en importancia, para que la relación estudiante y sujeto de cuidado se establezca en las mismas condiciones. Dado que la bioética permite la reflexión sobre las situaciones que posibilitan el cuidado y la interculturalidad se establece como un puente para acercar al futuro profesional y al sujeto de cuidado, reiterando que el primer camino es la reflexión pedagógica que reconstruya el currículo.

\section{CONCLUSIÓN}

La teorización surge como un modelo integrativo que recoge las interacciones discursivas y las organiza de manera lógica para explicar el fenómeno estudiado. De esta manera emerge la categoría central tejer la bioética intercultural que ante la colonización del ser y el saber en la formación bioética de futuros profesionales, plantea una reflexión pedagógica en los contextos interculturales que permita reconstruir el currículo para lograr flexibilidad en el mismo, abriendo paso a una visión más humana del cuidado. Cuyo origen es la decolonización del ser en la identidad profesional y el reconocimiento del cuidado como un acto compartido, alejándose de la visión técnica que plantea la responsabilidad como únicamente del profesional. Esto da paso a la de-colonización del saber, que se hace posible en la medida que hay comunicación entre el sujeto cuidado y el cuidador, esta comunicación va más allá de palabras, implica la escucha y el contacto con el otro, el aprendizaje desde el otro. Así, la de-colonización posibilita que el futuro profesional sea parte de una comunidad o contexto del otro, en un ejercicio que transite del aprendizaje a la comprensión, consolidando un currículo que se construye permanentemente desde el contexto y no para el contexto, señalando el rol del docente como fundamental. Lo anterior producto de la teoría fundamentada como un tipo de abordaje de investigación cualitativa que permitió describir los sentidos de la realidad de la formación bioética y así generar teoría propia a la disciplina de enfermería, al explorar otras formas de repensar la educación desde un contexto diverso, llevando a una articulación y coherencia entre el conocimiento y experiencia con rigor científico.

Agradecimientos. A la Universidad del Cauca, al Departamento de enfermería y al Hospital Mama Dominga por el apoyo brindado para la realización de la investigación. 


\section{REFERENCIAS}

Arraéz, J., Calles, J., \& Moreno, L. (2006). La hermenéutica una actividad interpretativa. Revista universitaria de investigación, 7(2), 171-181. Recuperado de: https://www.redalyc.org/articulo.oa?id=41070212

Blasco, J., \& Peréz, J. (2007). Metodologías de investigación en las ciencias de la actividad física y el deporte. Alicante: Club Universitario.

Campinha-Bacote, J. (2015). The Process of Cultural Competence in the Delivery of Healthcare Services: A Model of Care. Journal of Transcultural Nursing, 13(3), 181-184. doi: https://doi.org/10.1177/10459602013003003

Corbin, J., \& Strauss, A. (2015). Basics of Qualitative Research:Techniques and Procedures for Developing Grounded Theory(4 edition). Los Angeles: SAGE Publications

DANE. (2014). Censo Nacional Agropecuario. Bogotá: DANE.

De Souza, J. (2013). La pedagogía de la felicidad en una educación para la vida. En C. Walsh, Pedagogías decoloniales: Prácticas insurgentes para resistir (re)existir y (re)vivir (pp. 469-507). Ecuador: Abya Yala.

De Zubiría, S. (2016). Sentido social de la universidad: dilemas y potencialidades. En D. M. Rodríguez (Ed.), Bioética. Ecología de saberes. ¿La vida debe tener prioridades sobre los intereses de la ciencia? (pp. 3749). Bogotá: Universidad Libre.

Fawcett, J. (2005). Criteria for Evaluation of Theory. Nursing Science Quarterly, 18(2), 131 - 135.

Gadamer, H. (1993). Verdad y Método. (5 ed.). Salamanca: Sígueme.

Holzapfel, C. (2005). A la búsqueda del sentido. Revista de Filosofía, 163-166.

Jirwe, M., Gerrish, K., \& Emami, A. (2010). Student nurses' experiences of communication in cross-cultural care encounters. Scandinavian Journal of Caring Sciences, 24(1), 436-444. doi: 10.1111/j.14716712.2009.00733.x

Menéndez, E. (2017). Interculturalidad en salud. Dudas limitaciones y repliegues. En G. y. Martin, Medicinas, cuerpos en América Latina. Ecuador: Abya Yala.

Mignolo, W. (2007). El pensamiento decolonial: desprendimiento y apertura. En S. Castro y R. Grosfoguel (Eds.), El giro decolonial. Reflexiones para una diversidad epistémica más allá del capitalismo global (pp. 25-46). Bogotá: Siglo del Hombre Editores.

Niño, R. (2011). Metodología de la Investigación. Bogotá: Ediciones de la U.

Salgado, A. (2007). Investigación cualitativa: diseños, evaluación del rigor metodológico y retos. Revista Liberabit, 13(13), 71-78.

Sánchez, E. (2012). La investigación cualitativa en psicología: ¿Por qué esta metodología? Quaderns de psicología, 14 (1), 83-92.

Sánchez, G., \& Sánchez, R. (2010). Hermenéutica ¿nueva koiné?: ¿cómo reducir su relativización?. En J. M. (compilador), Hermenéutica en Acción. Cali: Editorial Universidad del Valle

Sánchez, G., Martín, C., \& Canal, B. (2017). La práctica de la investigación cualitativa: ejemplificación de estudios. Aveiro: Ludomedia.

Strauss, A., \& Corbin, J. (2002). Bases de la investigación cualitativa: técnicas y procedimientos para desarrollar la teoría fundamentada. Medellín: Universidad de Antioquia.

Valencia, V. (2010). De la escritura: punto de partida de la hermenéutica. En J. M. (compilador), Hermenéutica en Acción. Cali: Editorial Universidad del Valle. 